\title{
Author Correction: The nuclear export protein XPO1 — from biology to targeted therapy
}

Asfar S. Azmi (D, Mohammed H. Uddin (1) and Ramzi M. Mohammad

Correction to: Nature Reviews Clinical Oncology https://doi.org/10.1038/s41571-020-00442-4, published online 10 November 2020.

The author's original affiliation failed to include Karmanos Cancer Institute, which all three authors are affiliated with. This omission has been corrected in the online version of the manuscript.

https://doi.org/10.1038/s41571-020-00454-0 I Published online 24 November 2020

(C) Springer Nature Limited 2020 